\title{
CONVERGENCE OF ITERATIVE ALGORITHMS TO COMMON RANDOM FIXED POINTS OF RANDOM OPERATORS
}

\author{
ISMAT BEG AND MUJAHID ABBAS \\ Received 17 January 2006; Revised 27 June 2006; Accepted 6 July 2006 \\ This work is dedicated to Professor S. P. Singh on his 70th birthday
}

We prove the existence of a common random fixed point of two asymptotically nonexpansive random operators through strong and weak convergences of an iterative process. The necessary and sufficient condition for the convergence of sequence of measurable functions to a random fixed point of asymptotically quasi-nonexpansive random operators in uniformly convex Banach spaces is also established.

Copyright (C) 2006 I. Beg and M. Abbas. This is an open access article distributed under the Creative Commons Attribution License, which permits unrestricted use, distribution, and reproduction in any medium, provided the original work is properly cited.

\section{Introduction}

Random nonlinear analysis is an important mathematical discipline which is mainly concerned with the study of random nonlinear operators and their properties and is needed for the study of various classes of random equations. The study of random fixed point theory was initiated by the Prague school of probabilists in the 1950s [15, 16, 31]. Common random fixed point theorems are stochastic generalization of classical common fixed point theorems. The machinery of random fixed point theory provides a convenient way of modelling many problems arising from economic theory, see for example [27] and references mentioned therein. Random methods have revolutionized the financial markets. The survey article by Bharucha-Reid [10] attracted the attention of several mathematicians and gave wings to this theory. Itoh [18] extended Spacek's and Hans's theorem to multivalued contraction mappings. Now this theory has become the full fledged research area and various ideas associated with random fixed point theory are used to obtain the solution of nonlinear random system (see $[6,7,9,17,29]$ ). Papageorgiou $[25,26]$, Beg $[4,5]$ studied common random fixed points and random coincidence points of a pair of compatible random operators and proved fixed point theorems for contractive random operators in Polish spaces. Recently, Beg and Shahzad [8], Choudhury [11], and Badshah and Sayyed [3] used different iteration processes to obtain common random fixed points. The aim of this paper is to find common random fixed points of two asymptotically

Hindawi Publishing Corporation

Journal of Applied Mathematics and Stochastic Analysis

Volume 2006, Article ID 89213, Pages 1-16

DOI 10.1155/JAMSA/2006/89213 
nonexpansive random operators through strong as well as weak convergences of sequence of measurable functions in the setup of uniformly convex Banach spaces. We construct different random iterative algorithms for asymptotically quasi-nonexpansive random operators on an arbitrary Banach space and establish their convergence to random fixed point of the operators mentioned afore.

\section{Preliminaries}

Let $(\Omega, \Sigma)$ be a measurable space ( $\Sigma$-sigma algebra) and let $F$ be a nonempty subset of a Banach space $X$. We will denote by $C(X)$ the family of all compact subsets of $X$ with Hausdorff metric $H$ induced by the metric of $X$. A mapping $\xi: \Omega \rightarrow X$ is measurable if $\xi^{-1}(U) \in \Sigma$, for each open subset $U$ of $X$. The mapping $T: \Omega \times X \rightarrow X$ is a random map if and only if for each fixed $x \in X$, the mapping $T(\cdot, x): \Omega \rightarrow X$ is measurable and it is continuous if for each $\omega \in \Omega$, the mapping $T(\omega, \cdot): X \rightarrow X$ is continuous. A measurable mapping $\xi: \Omega \rightarrow X$ is a random fixed point of a random map $T: \Omega \times X \rightarrow X$ if and only if $T(\omega, \xi(\omega))=\xi(\omega)$, for each $\omega \in \Omega$. We denote the set of random fixed points of a random map $T$ by $\operatorname{RF}(T)$.

Let $B\left(x_{0}, r\right)$ denote the spherical ball centered at $x_{0}$ with radius $r$, defined as the set $\left\{x \in X:\left\|x-x_{0}\right\| \leq r\right\}$.

We denote the $n$th iterate $T(\omega, T(\omega, T(\omega, \ldots, T(\omega, x) \ldots)))$ of $T$ by $T^{n}(\omega, x)$. The letter $I$ denotes the random mapping $I: \Omega \times X \rightarrow X$ defined by $I(\omega, x)=x$ and $T^{0}=I$.

Definition 2.1. Let $F$ be a nonempty separable subset of a Banach space $X$ and let $T$ : $\Omega \times F \rightarrow F$ be a random map. The map $T$ is said to be the following.

(a) Asymptotically nonexpansive random operator if there exists a sequence of numbers $\left\{k_{n}\right\}$ (depending on $\omega$ ) in $[1, \infty)$ with $\lim _{n \rightarrow \infty} k_{n}=1$, where $n \in \mathbb{N}$ (set of natural numbers) such that for arbitrary $x, y \in F$,

$$
\left\|T^{n}(\omega, x)-T^{n}(\omega, y)\right\| \leq k_{n}\|x-y\|, \quad \text { for each } \omega \in \Omega .
$$

(b) Asymptotically quasi-nonexpansive random operator if for each $\omega \in \Omega, G(\omega)=$ $\{x \in F: x=T(\omega, x)\} \neq \phi$ and there exists a sequence of real numbers $\left\{k_{n}\right\}$ (depending on $\omega)$ in $[0, \infty)$ with $\lim _{n \rightarrow \infty} k_{n}=0$, where $n \in \mathbb{N}$ such that for $x \in F$ and $y \in G(\omega)$, the following inequality holds:

$$
\left\|T^{n}(\omega, x)-y\right\| \leq\left(1+k_{n}\right)\|x-y\|, \quad \text { for each } \omega \in \Omega .
$$

(c) Uniformly $k$-Lipschitzian random operator if for arbitrary $x, y \in F$,

$$
\left\|T^{n}(\omega, x)-T^{n}(\omega \cdot y)\right\| \leq k\|x-y\|
$$

where $n=1,2, \ldots$ and $k$ is a positive constant.

(d) Uniformly $(k-\alpha)$-Lipschitzian random operator if for arbitrary $x, y \in F$,

$$
\left\|T^{n}(\omega, x)-T^{n}(\omega \cdot y)\right\| \leq k\|x-y\|^{\alpha}, \quad \text { for each } \omega \in \Omega,
$$

where $n=1,2, \ldots, k$ is a positive constant, and $\alpha>0$. 
(e) Completely continuous random operator if for any sequence $\left\{x_{n}\right\}$ in $F$ such that $x_{n}$ converges weakly to $x, \lim _{n \rightarrow \infty}\left\|T\left(\omega, x_{n}\right)-T(\omega, x)\right\|=0$, for each $\omega \in \Omega$.

(f) Quasi-nonexpansive random operator if for each $\omega \in \Omega, G(\omega)=\{x \in F: x=T(\omega$, $x)\} \neq \phi$ and for any $x \in F$ and $y \in G(\omega)$,

$$
\|T(\omega, x)-y\| \leq\|x-y\|, \quad \text { for each } \omega \in \Omega
$$

Definition 2.2 (random Ishikawa iterative process). Let $T: \Omega \times F \rightarrow F$ be a random operator, where $F$ is a nonempty convex subset of a separable Banach space $X$. The random Ishikawa iterative process is the sequence of functions $\left\{\xi_{n}\right\}$ and $\left\{\eta_{n}\right\}$ defined by

$$
\begin{gathered}
\xi_{n+1}(\omega)=\left(1-\alpha_{n}\right) \xi_{n}(\omega)+\alpha_{n} T\left(\omega, \eta_{n}(\omega)\right), \\
\eta_{n}(\omega)=\left(1-\beta_{n}\right) \xi_{n}(\omega)+\beta_{n} T\left(\omega, \xi_{n}(\omega)\right), \quad \text { for each } \omega \in \Omega,
\end{gathered}
$$

$n=0,1,2, \ldots$, where $\left\{\alpha_{n}\right\}$ and $\left\{\beta_{n}\right\}$ are the sequences of real numbers in $[0,1]$ and $\xi_{0}$ : $\Omega \rightarrow F$ is an arbitrarily fixed measurable mapping. Obviously, $\left\{\xi_{n}\right\}$ and $\left\{\eta_{n}\right\}$ are sequences of functions from $\Omega$ to $F$.

Definition 2.3. Let $T, S: \Omega \times F \rightarrow F$ be two asymptotically nonexpansive random operators, where $F$ is a nonempty convex subset of a separable Banach space $X$. Consider a sequence of functions $\left\{\xi_{n}\right\}$ and $\left\{\eta_{n}\right\}$ defined by

$$
\begin{gathered}
\xi_{n+1}(\omega)=\left(1-\alpha_{n}\right) \xi_{n}(\omega)+\alpha_{n} S^{n}\left(\omega, \eta_{n}(\omega)\right), \\
\eta_{n}(\omega)=\left(1-\beta_{n}\right) \xi_{n}(\omega)+\beta_{n} T^{n}\left(\omega, \xi_{n}(\omega)\right),
\end{gathered}
$$

for each $\omega \in \Omega, n=0,1,2, \ldots$, where $\left\{\alpha_{n}\right\}$ and $\left\{\beta_{n}\right\}$ are sequences of real numbers in $[0,1]$ and $\xi_{0}: \Omega \rightarrow F$ is an arbitrarily fixed measurable mapping. Since $F$ is a convex set, it follows that for each $n, \xi_{n}$ is a mapping from $\Omega$ to $F$.

Remark 2.4. Let $F$ be a closed and convex subset of a separable Banach space $X$ and the sequence of functions $\left\{\xi_{n}\right\}$ defined as in Definition 2.3 is pointwise convergent, that is, $\xi_{n}(\omega) \rightarrow q:=\xi(\omega)$, for each $\omega \in \Omega$. Then closedness of $F$ implies that $\xi$ is a mapping from $\Omega$ to $F$. Since $F$ is a subset of a separable Banach space $X$, so if $T$ is a continuous random operator, then by $\left[2\right.$, Lemma 8.2.3], the map $\omega \rightarrow T^{n}(\omega, f(\omega))$ is a measurable function for any measurable function $f$ from $\Omega$ to $F$. Hence $\left\{\xi_{n}\right\}$ is a sequence of measurable functions and $\xi: \Omega \rightarrow F$ being a limit of a sequence of measurable functions is also measurable.

Definition 2.5. A Banach space $X$ is said to satisfy Opial's condition (Opial [24]). If sequence $\left\{x_{n}\right\}$ in $X$ converges weakly to $x \in X$ and $x \neq y$, then $\liminf _{n}\left\|x_{n}-y\right\|>\liminf _{n}$ $\left\|x_{n}-x\right\|$.

Banach spaces satisfying Opial's condition include Hilbert spaces and $l^{p}(1<p<\infty)$ spaces while $L_{p}$ spaces $(p \neq 2)$ are not Opial spaces.

Definition 2.6. Let $F$ be a nonempty bounded and convex subset of a Banach space $X$. A mapping $T: F \rightarrow X$ is called demiclosed with respect to $y \in X$ if for any sequence $\left\{x_{n}\right\}$ in 
$F$ the following implication holds: $w-\lim _{n \rightarrow \infty} x_{n}=x$ and $\lim _{n \rightarrow \infty}\left|T\left(x_{n}\right)-y\right|=0$ implies $x \in F$ and $T(x)=y$. For further details and other related results we refer to [1].

A random operator $T: \Omega \times F \rightarrow X$ is called demiclosed if $T(\omega, \cdot)$ is demiclosed, for each $\omega \in \Omega$.

LEMмa 2.7 (see [14]). Let $F$ be a nonempty closed bounded and convex subset of a uniformly convex separable Banach space X satisfying the Opial condition. Let $T$ be an asymptotically nonexpansive mapping of $F$ into itself. Then $I-T$ is demiclosed with respect to 0 .

Lemma 2.8 (see [28]). Suppose that $X$ is a uniformly convex Banach space and $0<p \leq \lambda_{n} \leq$ $q<1$ for all $n=1,2, \ldots$. Suppose $\left\{x_{n}\right\}$ and $\left\{y_{n}\right\}$ are sequences in $X$ such that $\limsup _{n \rightarrow \infty}\left\|x_{n}\right\| \leq r, \limsup _{n \rightarrow \infty}\left\|y_{n}\right\| \leq r$, and $\lim _{n \rightarrow \infty}\left\|\lambda_{n} x_{n}+\left(1-\lambda_{n}\right) y_{n}\right\|=r$ for some $r \geq 0$. Then $\lim _{n \rightarrow \infty}\left\|x_{n}-y_{n}\right\|=0$.

Lemma 2.9 (see [22, Lemma 2]). Let the nonnegative number sequences $\left\{\alpha_{n}\right\},\left\{\beta_{n}\right\}$, and $\left\{\gamma_{n}\right\}$ satisfy that

$$
\alpha_{n+1} \leq\left(1+\gamma_{n}\right) \alpha_{n}+\beta_{n}, \quad \text { for each } n=1,2, \ldots, \quad \sum_{n=1}^{\infty} \beta_{n}, \quad \sum_{n=1}^{\infty} \gamma_{n}<\infty .
$$

Then

(1) $\lim _{n \rightarrow \infty} \alpha_{n}$ exists,

(2) moreover, if $\lim _{n \rightarrow \infty}$ inf $\alpha_{n}=0$, then $\lim _{n \rightarrow \infty} \alpha_{n}=0$.

\section{Common random fixed point of asymptotically nonexpansive random operators}

In this section, we find common random fixed point of two asymptotically nonexpansive random operators. To achieve this purpose, we construct a sequence of measurable functions and consider its strong as well as weak convergences to a common random fixed point of random operators mentioned afore in the framework of uniformly convex Banach spaces. For the sake of completeness, we give the following random version of [19, Lemma 3].

THeOREm 3.1. Let $F$ be a nonempty closed, bounded, and convex subset of a normed space $X$ and let $T, S: \Omega \times F \rightarrow F$ be two uniformly $k$-Lipschitzian random contractive operators. Define the sequence of functions $\left\{\xi_{n}\right\}$ as in (2.7). If for each $\omega \in \Omega$,

$$
\lim _{n \rightarrow \infty}\left\|\xi_{n}(\omega)-S^{n}\left(\omega, \xi_{n}(\omega)\right)\right\|=0=\lim _{n \rightarrow \infty}\left\|\xi_{n}(\omega)-T^{n}\left(\omega, \xi_{n}(\omega)\right)\right\|
$$

then

$$
\lim _{n \rightarrow \infty}\left\|\xi_{n}(\omega)-S\left(\omega, \xi_{n}(\omega)\right)\right\|=0=\lim _{n \rightarrow \infty}\left\|\xi_{n}(\omega)-T\left(\omega, \xi_{n}(\omega)\right)\right\|,
$$

for each $\omega \in \Omega$.

Theorem 3.2. Let $F$ be a nonempty closed, bounded, and convex subset of a separable uniformly convex Banach space $X$ and let $T, S: \Omega \times F \rightarrow F$ be two continuous asymptotically nonexpansive random operators with sequence $\left\{k_{n}\right\}$ of real numbers in $[1, \infty)$ satisfying $\sum_{n=1}^{\infty}\left(k_{n}-1\right)<\infty$. Let the sequence of functions $\left\{\xi_{n}\right\}$ be as given in (2.7), where $\left\{\alpha_{n}\right\}$ and 
$\left\{\beta_{n}\right\}$ are the sequences of numbers in $[\varepsilon, 1-\varepsilon]$ for some $\varepsilon \in(0,1)$ and $\xi_{0}: \Omega \rightarrow F$ is an arbitrary measurable mapping. If $(I-T)(\omega, \cdot)$ and $(I-S)(\omega, \cdot)$ are demiclosed at 0 and the set $\{x \in F: I(\omega, x)-T(\omega, x)=0\} \cap\{x \in F: I(\omega, x)-S(\omega, x)=0\}$ is nonempty for each $\omega \in \Omega$, then

$$
\lim _{n \rightarrow \infty}\left\|S\left(\omega, \xi_{n}(\omega)\right)-\xi_{n}(\omega)\right\|=0=\lim _{n \rightarrow \infty}\left\|T\left(\omega, \xi_{n}(\omega)\right)-\xi_{n}(\omega)\right\|
$$

for each $\omega \in \Omega$.

Proof. For each $n$, define a mapping $f_{n}: \Omega \times F \rightarrow \mathbb{R}$ by

$$
f_{n}(\omega, x)=\|I(\omega, x)-T(\omega, x)\|+\|I(\omega, x)-S(\omega, x)\|-\frac{1}{n} .
$$

Set $F_{n}(\omega)=\left\{x \in F: f_{n}(\omega, x)<0\right\}, H_{n}(\omega)=\operatorname{cl}\left\{F_{n}(\omega): \omega \in \Omega\right\}$. Since $X$ is uniformly convex Banach space, therefore, $F$ is weakly compact so using similar arguments as given in [30, Theorem 3.1], we obtain a measurable selector $\zeta_{n}$ of $H_{n}$. Also for each $n$, define $L_{n}$ : $\Omega \rightarrow W K(F)$ by $L_{n}(\omega)=w-\operatorname{cl}\left\{\zeta_{i}(\omega): i \geq n\right\}$ and $L: \Omega \rightarrow W K(F)$ by $L(\omega)=\cap_{k \geq 1} w-$ $\operatorname{cl}\left\{\cup L_{n}(\omega): n \geq k\right\}$. We obtain a subsequence $\left\{\zeta_{k}(\omega)\right\}$ of $\left\{\zeta_{n}(\omega)\right\}$ which converges weakly to a measurable selector $\zeta(\omega)$ of $L$. Now for each $k,\left\{I\left(\omega, \zeta_{k}(\omega)\right)-T\left(\omega, \zeta_{k}(\omega)\right)\right\}$ and $\left\{I\left(\omega, \zeta_{k}(\omega)\right)-S\left(\omega, \zeta_{k}(\omega)\right)\right\}$ converge to 0 . Using demiclosedness of $(I-T)(\omega, \cdot)$ and $(I-$ $S)(\omega, \cdot)$ at 0 , we get $\zeta$ as a common random fixed point of $S$ and $T$. Consider

$$
\begin{aligned}
&\left\|\xi_{n+1}(\omega)-\zeta(\omega)\right\| \\
&=\left\|\left(1-\alpha_{n}\right) \xi_{n}(\omega)+\alpha_{n} S^{n}\left(\omega, \eta_{n}(\omega)\right)-\zeta(\omega)\right\| \\
& \leq \alpha_{n}\left\|S^{n}\left(\omega, \eta_{n}(\omega)\right)-\zeta(\omega)\right\|+\left(1-\alpha_{n}\right)\left\|\xi_{n}(\omega)-\zeta(\omega)\right\| \\
& \leq \alpha_{n} k_{n}\left\|\eta_{n}(\omega)-\zeta(\omega)\right\|+\left(1-\alpha_{n}\right)\left\|\xi_{n}(\omega)-\zeta(\omega)\right\| \\
&= \alpha_{n} k_{n}\left\|\left(1-\beta_{n}\right) \xi_{n}(\omega)+\beta_{n} T^{n}\left(\omega, \xi_{n}(\omega)\right)-\zeta(\omega)\right\|+\left(1-\alpha_{n}\right)\left\|\xi_{n}(\omega)-\zeta(\omega)\right\| \\
& \leq \alpha_{n} k_{n}\left[\beta_{n}\left\|T^{n}\left(\omega, \xi_{n}(\omega)\right)-\zeta(\omega)\right\|+\left(1-\beta_{n}\right)\left\|\xi_{n}(\omega)-\zeta(\omega)\right\|\right] \\
&+\left(1-\alpha_{n}\right)\left\|\xi_{n}(\omega)-\zeta(\omega)\right\| \\
& \leq \alpha_{n} k_{n}\left[\beta_{n} k_{n}\left\|\xi_{n}(\omega)-\zeta(\omega)\right\|+\left(1-\beta_{n}\right)\left\|\xi_{n}(\omega)-\zeta(\omega)\right\|\right]+\left(1-\alpha_{n}\right)\left\|\xi_{n}(\omega)-\zeta(\omega)\right\| \\
&= {\left[\left(1-\alpha_{n}\right)+\alpha_{n} \beta_{n} k_{n}^{2}+\alpha_{n} k_{n}\left(1-\beta_{n}\right)\right]\left\|\xi_{n}(\omega)-\zeta(\omega)\right\|, }
\end{aligned}
$$

for each $\omega \in \Omega$. Define $v_{n}=\left(1-\alpha_{n}\right)+\alpha_{n} \beta_{n} k_{n}^{2}+\alpha_{n} k_{n}\left(1-\beta_{n}\right)$. Then

$$
\left\|\xi_{n+1}(\omega)-\zeta(\omega)\right\| \leq v_{n}\left\|\xi_{n}(\omega)-\zeta(\omega)\right\|, \quad \text { for each } \omega \in \Omega
$$

and for each $n=1,2, \ldots$. Consequently, we have

$$
\left\|\xi_{n+m}(\omega)-\zeta(\omega)\right\| \leq\left(\prod_{i=n}^{n+m-1} v_{i}\right)\left\|\xi_{n}(\omega)-\zeta(\omega)\right\|, \quad \text { for each } \omega \in \Omega
$$


Also we have $\sum_{n=1}^{\infty}\left(v_{n}-1\right)<\infty$, thus $\lim _{n \rightarrow \infty} \prod_{i=n}^{\infty} v_{i}$ exists and hence $\lim _{n \rightarrow \infty} \| \xi_{n}(\omega)-$ $\zeta(\omega) \|$ exists, for each $\omega \in \Omega$. Now if $\lim _{n \rightarrow \infty}\left\|\xi_{n}(\omega)-\zeta(\omega)\right\|=0$, for each $\omega \in \Omega$, then the result follows. Suppose $\lim _{n \rightarrow \infty}\left\|\xi_{n}(\omega)-\zeta(\omega)\right\|=c$, where $c>0$, for each $\omega \in \Omega$. As

$$
\left\|T^{n}\left(\omega, \xi_{n}(\omega)\right)-\zeta(\omega)\right\| \leq k_{n}\left\|\xi_{n}(\omega)-\zeta(\omega)\right\|, \quad \text { for each } \omega \in \Omega,
$$

where $n=1,2, \ldots$, so $\limsup _{n \rightarrow \infty}\left\|T^{n}\left(\omega, \xi_{n}(\omega)\right)-\zeta(\omega)\right\| \leq c$, for each $\omega \in \Omega$. Now consider

$$
\begin{aligned}
\left\|\eta_{n}(\omega)-\zeta(\omega)\right\| & =\left\|\left(1-\beta_{n}\right) \xi_{n}(\omega)+\beta_{n} T^{n}\left(\omega, \xi_{n}(\omega)\right)-\zeta(\omega)\right\| \\
& \leq \beta_{n}\left\|T^{n}\left(\omega, \xi_{n}(\omega)\right)-\zeta(\omega)\right\|+\left(1-\beta_{n}\right)\left\|\xi_{n}(\omega)-\zeta(\omega)\right\| \\
& \leq \beta_{n} k_{n}\left\|\xi_{n}(\omega)-\zeta(\omega)\right\|+\left(1-\beta_{n}\right)\left\|\xi_{n}(\omega)-\zeta(\omega)\right\| \\
& =\left\|\xi_{n}(\omega)-\zeta(\omega)\right\|+\left(k_{n}-1\right) \beta_{n}\left\|\xi_{n}(\omega)-\zeta(\omega)\right\| \\
& \leq\left\|\xi_{n}(\omega)-\zeta(\omega)\right\|+\left(k_{n}-1\right)(1-\varepsilon)\left\|\xi_{n}(\omega)-\zeta(\omega)\right\|
\end{aligned}
$$

for each $\omega \in \Omega$. Hence

$$
\lim \sup _{n \rightarrow \infty}\left\|\eta_{n}(\omega)-\zeta(\omega)\right\| \leq c
$$

Now

$$
\left\|S^{n}\left(\omega, \eta_{n}(\omega)\right)-\zeta(\omega)\right\| \leq k_{n}\left\|\eta_{n}(\omega)-\zeta(\omega)\right\|, \quad \text { for each } \omega \in \Omega .
$$

Now $k_{n} \rightarrow 1$ as $n \rightarrow \infty$ and (3.10) give

$$
\lim \sup _{n \rightarrow \infty}\left\|S^{n}\left(\omega, \eta_{n}(\omega)\right)-\zeta(\omega)\right\| \leq c, \quad \text { for each } \omega \in \Omega
$$

Hence

$$
\begin{aligned}
\lim _{n \rightarrow \infty} \| & \left\|\xi_{n+1}(\omega)-\zeta(\omega)\right\| \\
& =\lim _{n \rightarrow \infty}\left\|\left(1-\alpha_{n}\right) \xi_{n}(\omega)+\alpha_{n} S^{n}\left(\omega, \eta_{n}(\omega)\right)-\zeta(\omega)\right\| \\
& =\lim _{n \rightarrow \infty}\left\|\left(1-\alpha_{n}\right)\left(\xi_{n}(\omega)-\zeta(\omega)\right)+\alpha_{n}\left(S^{n}\left(\omega, \eta_{n}(\omega)\right)-\zeta(\omega)\right)\right\|=c,
\end{aligned}
$$

for each $\omega \in \Omega$. Applying Lemma 2.8, we have

$$
\lim _{n \rightarrow \infty}\left\|S^{n}\left(\omega, \eta_{n}(\omega)\right)-\xi_{n}(\omega)\right\|=0, \quad \text { for each } \omega \in \Omega .
$$

Now

$$
\begin{aligned}
\| \xi_{n}(\omega) & -\zeta(\omega) \| \\
\leq & \left\|\xi_{n}(\omega)-S^{n}\left(\omega, \eta_{n}(\omega)\right)\right\|+\left\|S^{n}\left(\omega, \eta_{n}(\omega)\right)-\zeta(\omega)\right\| \\
\leq & \left\|\xi_{n}(\omega)-S^{n}\left(\omega, \eta_{n}(\omega)\right)\right\|+k_{n}\left\|\eta_{n}(\omega)-\zeta(\omega)\right\| .
\end{aligned}
$$

It implies that $c \leq \liminf _{n \rightarrow \infty}\left\|\eta_{n}(\omega)-\zeta(\omega)\right\|$, for each $\omega \in \Omega$. 
Now using (3.10), we have $\lim _{n \rightarrow \infty}\left\|\eta_{n}(\omega)-\zeta(\omega)\right\|=c$, for every $\omega \in \Omega$. That is,

$$
\begin{aligned}
\lim _{n \rightarrow \infty} & \left\|\left(1-\beta_{n}\right) \xi_{n}(\omega)+\beta_{n} T^{n}\left(\omega, \xi_{n}(\omega)\right)-\zeta(\omega)\right\| \\
& =\lim _{n \rightarrow \infty}\left\|\left(1-\beta_{n}\right)\left(\xi_{n}(\omega)-\zeta(\omega)\right)+\beta_{n}\left(T^{n}\left(\omega, \xi_{n}(\omega)\right)-\zeta(\omega)\right)\right\|=c,
\end{aligned}
$$

for each $\omega \in \Omega$. Hence by Lemma 2.8, we obtain

$$
\lim _{n \rightarrow \infty}\left\|T^{n}\left(\omega, \xi_{n}(\omega)\right)-\xi_{n}(\omega)\right\|=0
$$

for each $\omega \in \Omega$. Now

$$
\begin{aligned}
\left\|S^{n}\left(\omega, \xi_{n}(\omega)\right)-\xi_{n}(\omega)\right\| \\
\quad \leq\left\|S^{n}\left(\omega, \xi_{n}(\omega)\right)-S^{n}\left(\omega, \eta_{n}(\omega)\right)\right\|+\left\|S^{n}\left(\omega, \eta_{n}(\omega)\right)-\xi_{n}(\omega)\right\| \\
\quad \leq k_{n}\left\|\xi_{n}(\omega)-\eta_{n}(\omega)\right\|+\left\|S^{n}\left(\omega, \eta_{n}(\omega)\right)-\xi_{n}(\omega)\right\| \\
\quad \leq k_{n}\left\|\xi_{n}(\omega)-\left(\left(1-\beta_{n}\right) \xi_{n}(\omega)+\beta_{n} T^{n}\left(\omega, \xi_{n}(\omega)\right)\right)\right\|+\left\|S^{n}\left(\omega, \eta_{n}(\omega)\right)-\xi_{n}(\omega)\right\| \\
\quad \leq k_{n} \beta_{n}\left\|\xi_{n}(\omega)-T^{n}\left(\omega, \xi_{n}(\omega)\right)\right\|+\left\|S^{n}\left(\omega, \eta_{n}(\omega)\right)-\xi_{n}(\omega)\right\| \\
\quad \leq k_{n}(1-\varepsilon)\left\|\xi_{n}(\omega)-T^{n}\left(\omega, \xi_{n}(\omega)\right)\right\|+\left\|S^{n}\left(\omega, \eta_{n}(\omega)\right)-\xi_{n}(\omega)\right\|,
\end{aligned}
$$

for each $\omega \in \Omega$. So we have $\lim _{n \rightarrow \infty}\left\|S^{n}\left(\omega, \xi_{n}(\omega)\right)-\xi_{n}(\omega)\right\|=0$, for every $\omega \in \Omega$. Theorem 3.1 further gives

$$
\lim _{n \rightarrow \infty}\left\|\xi_{n}(\omega)-S\left(\omega, \xi_{n}(\omega)\right)\right\|=0=\lim _{n \rightarrow \infty}\left\|\xi_{n}(\omega)-T\left(\omega, \xi_{n}(\omega)\right)\right\|
$$

for each $\omega \in \Omega$.

Theorem 3.3. Let $F$ be a nonempty closed, bounded, and convex subset of a uniformly convex separable Banach space $X$ satisfying Opial's condition and let $T, S: \Omega \times F \rightarrow F$ and the sequence of functions $\left\{\xi_{n}\right\}$ be as taken in Theorem 3.2, moreover, if $S(\omega, \cdot)$ and $T(\omega, \cdot)$ are completely continuous and $\{x \in F: I(\omega, x)-T(\omega, x)=0\} \cap\{x \in F: I(\omega, x)-S(\omega, x)=$ $0\}$ is nonempty, for each $\omega \in \Omega$, then $S$ and $T$ have a common random fixed point and $\left\{\xi_{n}\right\}$ converges weakly to a common random fixed point of $S$ and $T$.

Proof. Following the similar argument as used in Theorem 3.2, Let $\zeta: \Omega \rightarrow F$ be the common random fixed point of $S$ and T. Let $\phi$ and $\varphi$ be two functions from $\Omega$ to $F$ and let $\left\{\xi_{m}(\omega)\right\}$ and $\left\{\xi_{k}(\omega)\right\}$ be two subsequences of $\left\{\xi_{n}(\omega)\right\}$ such that $\left\{\xi_{m}(\omega)\right\}$ and $\left\{\xi_{k}(\omega)\right\}$ converge weakly to $\phi(\omega)$ and $\varphi(\omega)$, for each $\omega \in \Omega$ as $m, k \rightarrow \infty$. As proved in Theorem 3.2, $\lim _{n \rightarrow \infty}\left\|\xi_{n}(\omega)-\zeta(\omega)\right\|$ exists, for each $\omega \in \Omega$. Now by $\lim _{n \rightarrow \infty}\left\|\xi_{n}(\omega)-S\left(\omega, \xi_{n}(\omega)\right)\right\|=$ $0=\lim _{n \rightarrow \infty}\left\|\xi_{n}(\omega)-S\left(\omega, \xi_{n}(\omega)\right)\right\|$, for each $\omega \in \Omega$ and $S$ and $T$ are asymptotically nonexpansive, $I-S(\omega, \cdot)$ and $I-T(\omega, \cdot)$ are demiclosed with respect to "0," by Lemma 2.7, it follows that $S(\omega, \phi(\omega))=\phi(\omega)$ and $T(\omega, \phi(\omega))=\phi(\omega)$. Now measurability of $\phi$ follows from the fact that $T$ and $S$ are completely continuous random operators and from Remark 2.4. Thus $\phi$ is a common random fixed point of $T$ and $S$. Similarly, it can be 
shown that $\varphi$ is also a common random fixed point of random operators $T$ and $S$. Now we prove that $\phi(\omega)=\varphi(\omega)$, for every $\omega \in \Omega$. If not so, then for some $\omega \in \Omega, \phi(\omega) \neq \varphi(\omega)$. Now by Opial's condition

$$
\begin{aligned}
\lim _{n \rightarrow \infty}\left\|\xi_{n}(\omega)-\phi(\omega)\right\| & =\lim _{m \rightarrow \infty}\left\|\xi_{m}(\omega)-\phi(\omega)\right\|<\lim _{m \rightarrow \infty}\left\|\xi_{m}(\omega)-\varphi(\omega)\right\| \\
& =\lim _{n \rightarrow \infty}\left\|\xi_{n}(\omega)-\varphi(\omega)\right\|=\lim _{k \rightarrow \infty}\left\|\xi_{k}(\omega)-\varphi(\omega)\right\| \\
& <\lim _{k \rightarrow \infty}\left\|\xi_{k}(\omega)-\phi(\omega)\right\|=\lim _{n \rightarrow \infty}\left\|\xi_{n}(\omega)-\phi(\omega)\right\| .
\end{aligned}
$$

This contradiction concludes the proof.

TheORem 3.4. Let $F$ be a nonempty compact and convex subset of a uniformly convex separable Banach space $X$ and let T, $S: \Omega \times F \rightarrow F$ and the sequence of functions $\left\{\xi_{n}\right\}$ be as taken in Theorem 3.2. Then $S$ and $T$ have a common random fixed point and $\left\{\xi_{n}\right\}$ converges to a common random fixed point of $S$ and $T$.

Proof. Since

$$
\lim _{n \rightarrow \infty}\left\|\xi_{n}(\omega)-S\left(\omega, \xi_{n}(\omega)\right)\right\|=0=\lim _{n \rightarrow \infty}\left\|\xi_{n}(\omega)-T\left(\omega, \xi_{n}(\omega)\right)\right\|
$$

for each $\omega \in \Omega$. As $F$ is compact, for each $n$, define $G_{n}: \Omega \rightarrow C(X)$ by $G_{n}(\omega)=\operatorname{cl}\left\{\xi_{i}(\omega)\right.$ : $i \geq n\}$, where cl denotes the closure. Define $G: \Omega \rightarrow C(X)$ by $G(\omega)=\bigcap_{n=1}^{\infty} G_{n}(\omega)$. Then $G$ is measurable and has a measurable selector $\xi$ [31]. We may assume that there exists a subsequence $\left\{\xi_{n_{j}}(\omega)\right\}$ of $\left\{\xi_{n}(\omega)\right\}$ such that $\xi_{n_{j}}(\omega) \rightarrow q(\omega)$ as $n_{j} \rightarrow \infty$, for each $\omega \in \Omega$. Also, continuity of $S$ and $T$ gives $S\left(\omega, \xi_{n_{j}}(\omega)\right) \rightarrow q(\omega)$ and $T\left(\omega, \xi_{n_{j}}(\omega)\right) \rightarrow q(\omega)$ as $n_{j} \rightarrow$ $\infty$, for each $\omega \in \Omega$. Now (3.21) gives $S(\omega, q(\omega))=q(\omega)=T(\omega, q(\omega))$, for each $\omega \in \Omega$. Hence $q: \Omega \rightarrow F$ is a common random fixed point of $S$ and $T$. Since $\lim _{n \rightarrow \infty} \| \xi_{n}(\omega)-$ $\zeta(\omega) \|$ exists for any common random fixed point $\zeta: \Omega \rightarrow F$ of random operators $S$ and $T$. Therefore, $\left\{\xi_{n}\right\}$ must itself converge to a common random fixed point $q: \Omega \rightarrow F$ of random operators $S$ and $T$.

\section{Convergence of random iterative scheme}

The term quasi-nonexpansiveness was first coined by Diaz and Metcalf [13]. Das et al. [12], Liu [21] also studied iterative schemes for quasi-nonexpansive mappings, Liu [20] and Liu [23] studied iteration process for different mappings with errors in Banach spaces. We present in this section a random iterative scheme for asymptotically quasi-nonexpansive random operator. We also establish the necessary and sufficient condition for the convergence of this scheme to a random fixed point of the random operator stated afore in the framework of Banach spaces.

THeOREM 4.1. Let F be a nonempty weakly compact and convex subset of a separable Banach space $X$, let $T: \Omega \times F \rightarrow F$ be a continuous asymptotically quasi-nonexpansive random operator with $\sum_{n=1}^{\infty} k_{n}<\infty$ and $(I-T)(\omega, \cdot)$ is demiclosed at zero, and the set $\{x \in F$ : $I(\omega, x)-T(\omega, x)=0\}$ is nonempty, for each $\omega \in \Omega$. Let $\xi_{0}: \Omega \rightarrow F$ be any fixed measurable 
mapping. Define the sequence of measurable functions $\left\{\xi_{n}\right\},\left\{\eta_{n}\right\},\left\{\varphi_{n}\right\}$, and $\left\{\zeta_{n}\right\}$ from $\Omega$ to $F$ as

$$
\begin{gathered}
\xi_{n+1}(\omega)=\alpha_{n} \xi_{n}(\omega)+\beta_{n} T^{n}\left(\omega, \eta_{n}(\omega)\right)+\gamma_{n} \varphi_{n}(\omega), \\
\eta_{n}(\omega)=\overline{\alpha_{n}} \xi_{n}(\omega)+\overline{\beta_{n}} T^{n}\left(\omega, \xi_{n}(\omega)\right)+\overline{\gamma_{n}} \zeta_{n}(\omega),
\end{gathered}
$$

where the sequences of numbers $\left\{\alpha_{n}\right\},\left\{\beta_{n}\right\},\left\{\gamma_{n}\right\},\left\{\overline{\alpha_{n}}\right\},\left\{\overline{\beta_{n}}\right\}$, and $\left\{\overline{\gamma_{n}}\right\}$ are in $[0,1]$ satisfying $\alpha_{n}+\beta_{n}+\gamma_{n}=\overline{\alpha_{n}}+\overline{\beta_{n}}+\overline{\gamma_{n}}=1, \sum_{n=1}^{\infty} \gamma_{n}$, and $\sum_{n=1}^{\infty} \overline{\gamma_{n}}<\infty$, for $n=1,2, \ldots$ Also if $\left\{\left\|\varphi_{n}(\omega)\right\|\right\}$ and $\left\{\left\|\zeta_{n}(\omega)\right\|\right\}$ are bounded, for every $\omega \in \Omega$, then $\lim _{n \rightarrow \infty}\left\|\xi_{n}(\omega)-\eta(\omega)\right\|=0$ for some $\eta \in \operatorname{RF}(T)$ if and only if $\lim _{n \rightarrow \infty} \inf \operatorname{dist}\left(\xi_{n}(\omega),\{\xi(\omega): \xi \in \operatorname{RF}(T)\}\right)=0$, for each $\omega \in \Omega$.

Proof. We note that $\mathrm{RF}(T)$ is nonempty by [30, Theorem 3.1]. Now if for some $\xi \in$ $\operatorname{RF}(T), \lim _{n \rightarrow \infty}\left\|\xi_{n}(\omega)-\xi(\omega)\right\|=0$, for each $\omega \in \Omega$ then obviously $\lim _{n \rightarrow \infty} \inf \operatorname{dist}\left(\xi_{n}(\omega)\right.$, $\{\xi(\omega): \xi \in \operatorname{RF}(T)\})=0$, for every $\omega \in \Omega$. Now for any $\xi \in \operatorname{RF}(T)$,

$$
\begin{aligned}
& \left\|\xi_{n+1}(\omega)-\xi(\omega)\right\| \\
& \quad=\left\|\alpha_{n} \xi_{n}(\omega)+\beta_{n} T^{n}\left(\omega, \eta_{n}(\omega)\right)+\gamma_{n} \varphi_{n}(\omega)-\xi(\omega)\right\| \\
& \quad \leq \alpha_{n}\left\|\xi_{n}(\omega)-\xi(\omega)\right\|+\beta_{n}\left\|T^{n}\left(\omega, \eta_{n}(\omega)\right)-\xi(\omega)\right\|+\gamma_{n}\left\|\varphi_{n}(\omega)-\xi(\omega)\right\| \\
& \quad \leq \alpha_{n}\left\|\xi_{n}(\omega)-\xi(\omega)\right\|+\beta_{n}\left(1+k_{n}\right)\left\|\eta_{n}(\omega)-\xi(\omega)\right\|+\gamma_{n}\left\|\varphi_{n}(\omega)-\xi(\omega)\right\|, \\
& \left\|\eta_{n}(\omega)-\xi(\omega)\right\| \\
& \quad=\left\|\overline{\alpha_{n}} \xi_{n}(\omega)+\overline{\beta_{n}} T^{n}\left(\omega, \xi_{n}(\omega)\right)+\overline{\gamma_{n}} \zeta_{n}(\omega)-\xi(\omega)\right\| \\
& \quad \leq \overline{\alpha_{n}}\left\|\xi_{n}(\omega)-\xi(\omega)\right\|+\overline{\beta_{n}}\left(1+k_{n}\right)\left\|\xi_{n}(\omega)-\xi(\omega)\right\|+\overline{\gamma_{n}}\left\|\zeta_{n}(\omega)-\xi(\omega)\right\| .
\end{aligned}
$$

Now using (4.3) in (4.2), we have

$$
\begin{aligned}
&\left\|\xi_{n+1}(\omega)-\xi(\omega)\right\| \\
& \leq \alpha_{n}\left\|\xi_{n}(\omega)-\xi(\omega)\right\|+\beta_{n}\left(1+k_{n}\right) \overline{\alpha_{n}}\left\|\xi_{n}(\omega)-\xi(\omega)\right\|+\beta_{n} \overline{\beta_{n}}\left(1+k_{n}\right)^{2}\left\|\xi_{n}(\omega)-\xi(\omega)\right\| \\
&+\overline{\gamma_{n}} \beta_{n}\left(1+k_{n}\right)\left\|\zeta_{n}(\omega)-\xi(\omega)\right\|+\gamma_{n}\left\|\varphi_{n}(\omega)-\xi(\omega)\right\| \\
&= \alpha_{n}\left\|\xi_{n}(\omega)-\xi(\omega)\right\|+\left(1-\alpha_{n}-\gamma_{n}\right)\left(1+k_{n}\right) \overline{\alpha_{n}}\left\|\xi_{n}(\omega)-\xi(\omega)\right\| \\
&+\overline{\beta_{n}}\left(1-\alpha_{n}-\gamma_{n}\right)\left(1+k_{n}\right)^{2}\left\|\xi_{n}(\omega)-\xi(\omega)\right\|+m_{n}(\omega) \\
& \leq \alpha_{n}\left\|\xi_{n}(\omega)-\xi(\omega)\right\|+\left(1-\alpha_{n}\right)\left(1+k_{n}\right)^{2} \overline{\alpha_{n}}\left\|\xi_{n}(\omega)-\xi(\omega)\right\| \\
&+\overline{\beta_{n}}\left(1-\alpha_{n}\right)\left(1+k_{n}\right)^{2}\left\|\xi_{n}(\omega)-\xi(\omega)\right\|+m_{n}(\omega) \\
& \leq \alpha_{n}\left(1+k_{n}\right)^{2}\left\|\xi_{n}(\omega)-\xi(\omega)\right\|+\left(1-\alpha_{n}\right)\left(1+k_{n}\right)^{2}\left(\overline{\alpha_{n}}+\overline{\beta_{n}}\right)\left\|\xi_{n}(\omega)-\xi(\omega)\right\|+m_{n}(\omega) \\
& \leq \alpha_{n}\left(1+k_{n}\right)^{2}\left\|\xi_{n}(\omega)-\xi(\omega)\right\|+\left(1-\alpha_{n}\right)\left(1+k_{n}\right)^{2}\left\|\xi_{n}(\omega)-\xi(\omega)\right\|+m_{n}(\omega) \\
&=\left(1+k_{n}\right)^{2}\left\|\xi_{n}(\omega)-\xi(\omega)\right\|+m_{n}(\omega),
\end{aligned}
$$


for every $\omega \in \Omega$, where $m_{n}: \Omega \rightarrow \mathbb{R}$ is given by

$$
m_{n}(\omega)=\beta_{n}\left(1+k_{n}\right) \overline{\gamma_{n}}\left\|\zeta_{n}(\omega)-\xi(\omega)\right\|+\gamma_{n}\left\|\varphi_{n}(\omega)-\xi(\omega)\right\|
$$

Thus for every $\omega \in \Omega$ and $\xi \in \operatorname{RF}(T)$, we have

$$
\left\|\xi_{n+1}(\omega)-\xi(\omega)\right\| \leq\left(1+k_{n}\right)^{2}\left\|\xi_{n}(\omega)-\xi(\omega)\right\|+m_{n}(\omega)
$$

Since $\sum_{n=1}^{\infty} \gamma_{n}$ and $\sum_{n=1}^{\infty} \overline{\gamma_{n}}<\infty$. Also, $\sum_{n=1}^{\infty} k_{n}<\infty\left\{\left\|\varphi_{n}(\omega)\right\|\right\}$ and $\left\{\left\|\zeta_{n}(\omega)\right\|\right\}$ are bounded, for every $\omega \in \Omega$. Thus, $\sum_{n=1}^{\infty} m_{n}(\omega)<\infty$, for every $\omega \in \Omega$. Now using (4.6), we have

$$
\operatorname{dist}\left(\xi_{n+1}(\omega), \operatorname{RF}(T)\right) \leq\left(1+k_{n}\right)^{2} \operatorname{dist}\left(\xi_{n}(\omega), \operatorname{RF}(T)\right)+m_{n}(\omega),
$$

for every $\omega \in \Omega$. Now Lemma 2.9 and $\lim _{n \rightarrow \infty} \inf \operatorname{dist}\left(\xi_{n}(\omega),\{\xi(\omega): \xi \in \operatorname{RF}(T)\}\right)=0$ imply $\lim _{n \rightarrow \infty} \operatorname{dist}\left(\xi_{n}(\omega),\{\xi(\omega): \xi \in \operatorname{RF}(T)\}\right)=0$, for every $\omega \in \Omega$. Now further using (4.6), we have

$$
\begin{aligned}
&\left\|\xi_{n+m}(\omega)-\xi(\omega)\right\| \\
& \leq\left(1+k_{n+m-1}\right)^{2}\left\|\xi_{n+m-1}(\omega)-\xi(\omega)\right\|+m_{n+m-1}(\omega) \\
& \leq \exp \left(2 k_{n+m-1}\right)\left\|\xi_{n+m-1}(\omega)-\xi(\omega)\right\|+m_{n+m-1}(\omega) \\
& \leq \exp \left(2 k_{n+m-1}\right) \exp \left(2 k_{n+m-2}\right)\left\|\xi_{n+m-2}(\omega)-\xi(\omega)\right\| \\
&+\exp \left(2 k_{n+m-1}\right) m_{n+m-2}(\omega)+m_{n+m-1}(\omega) \\
& \leq \exp 2\left(k_{n+m-1}\right)+\left(k_{n+m-2}\right)\left\|\xi_{n+m-2}(\omega)-\xi(\omega)\right\| \\
&+\exp \left(2 k_{n+m-1}\right)\left[m_{n+m-2}(\omega)+m_{n+m-1}(\omega)\right] \\
& \vdots \\
& \leq \exp \left(2 \sum_{j=n}^{n+m-1} k_{j}\right)\left\|\xi_{n}(\omega)-\xi(\omega)\right\|+\exp \left(2 \sum_{j=n}^{n+m-1} k_{j}\right)_{j=n}^{n+m-1} m_{j}(\omega) \\
& \leq M\left\|\xi_{n}(\omega)-\xi(\omega)\right\|+M \sum_{j=n}^{n+m-1} m_{j}(\omega) .
\end{aligned}
$$

That is,

$$
\left\|\xi_{n+m}(\omega)-\xi(\omega)\right\| \leq M\left\|\xi_{n}(\omega)-\xi(\omega)\right\|+M \sum_{j=n}^{n+m-1} m_{j}(\omega)
$$

for every $\omega \in \Omega$ and $m, n \in \mathbb{N}$, where $M=\exp \left(2 \sum_{j=n}^{\infty} k_{j}\right)>0$, for each $\omega \in \Omega$. Since $\lim _{n \rightarrow \infty} \operatorname{dist}\left(\xi_{n}(\omega),\{\xi(\omega): \xi \in \operatorname{RF}(T)\}\right)=0$ and $\sum_{n=1}^{\infty} m_{n}(\omega)<\infty$, for every $\omega \in \Omega$. Take 
$\varepsilon>0$, there exists $n_{1} \in \mathbb{N}$ such that $\operatorname{dist}\left(\xi_{n}(\omega),\{\xi(\omega): \xi \in \mathrm{RF}(T)\}\right)<\varepsilon / 3 M$ and $\sum_{k=n}^{n+m-1} m_{k}(\omega)<$ $\varepsilon / 3 M$, for each $\omega \in \Omega$ and $n \geq n_{1}$. So, there exists $\xi^{*} \in \operatorname{RF}(T)\left\|\xi_{n}(\omega)-\xi^{*}(\omega)\right\|<\varepsilon / 3 M$, for every $\omega \in \Omega$ and $n \geq n_{1}$. Consider for $n \geq n_{1}$ and $\omega \in \Omega$,

$$
\begin{aligned}
& \left\|\xi_{n+m}(\omega)-\xi_{n}(\omega)\right\| \\
& \quad \leq\left\|\xi_{n+m}(\omega)-\xi^{*}(\omega)\right\|+\left\|\xi^{*}(\omega)-\xi_{n}(\omega)\right\| \\
& \quad \leq M\left\|\xi_{n}(\omega)-\xi(\omega)\right\|+M \sum_{j=n}^{n+m-1} m_{j}(\omega)+M(\omega)\left\|\xi^{*}(\omega)-\xi_{n}(\omega)\right\| \\
& \quad<M \frac{\varepsilon}{3 M}+M \frac{\varepsilon}{3 M}+M \frac{\varepsilon}{3 M}=\varepsilon .
\end{aligned}
$$

It implies that $\left\{\xi_{n}(\omega)\right\}$ is a Cauchy sequence, for every $\omega \in \Omega$. Therefore, $\xi_{n}(\omega) \rightarrow p(\omega)$, for every $\omega \in \Omega, p: \Omega \rightarrow F$ being the limit of sequence of measurable functions is also measurable. There exist $n_{2}, n_{3} \in \mathbb{N}$ such that for $n \geq n_{2}, n_{3}$ and $\xi^{* *} \in \operatorname{RF}(T)$, we have $\left\|\xi_{n}(\omega)-p(\omega)\right\|<\varepsilon / 2\left(2+k_{1}\right)$ and $\left\|\xi_{n}(\omega)-\xi^{* *}(\omega)\right\|<\varepsilon / 2\left(4+3 k_{1}\right)$, for each $\omega \in \Omega$. Put $n_{4}=\max \left\{n_{2}, n_{3}\right\}$. Now

$$
\begin{aligned}
&\|T(\omega, p(\omega))-p(\omega)\| \\
& \leq\left\|T(\omega, p(\omega))-\xi^{* *}(\omega)\right\|+2\left\|T\left(\omega, \xi_{n_{4}}(\omega)\right)-\xi^{* *}(\omega)\right\| \\
&+\left\|\xi_{n_{4}}(\omega)-\xi^{* *}(\omega)\right\|+\left\|\xi_{n_{4}}(\omega)-p(\omega)\right\| \\
& \leq\left(1+k_{n}\right)\left\|p(\omega)-\xi^{* *}(\omega)\right\|+2\left(1+k_{n}\right)\left\|\xi_{n_{4}}(\omega)-\xi^{* *}(\omega)\right\| \\
&+\left\|\xi_{n_{4}}(\omega)-\xi^{* *}(\omega)\right\|+\left\|\xi_{n_{4}}(\omega)-p(\omega)\right\| \\
& \leq\left(1+k_{n}\right)\left\|\xi_{n_{4}}(\omega)-\xi^{* *}(\omega)\right\|+\left(1+k_{n}\right)\left\|p(\omega)-\xi_{n_{4}}(\omega)\right\| \\
&+2\left(1+k_{n}\right)\left\|\xi_{n_{4}}(\omega)-\xi^{* *}(\omega)\right\|+\left\|\xi_{n_{4}}(\omega)-\xi^{* *}(\omega)\right\|+\left\|\xi_{n_{4}}(\omega)-p(\omega)\right\| \\
& \leq\left(2+k_{n}\right)\left\|p(\omega)-\xi_{n_{4}}(\omega)\right\|+\left(4+3 k_{n}\right)\left\|\xi_{n_{4}}(\omega)-\xi^{* *}(\omega)\right\| \\
&<\left(2+k_{n}\right) \frac{\varepsilon}{2\left(2+k_{1}\right)}+\left(4+3 k_{n}\right) \frac{\varepsilon}{2\left(4+3 k_{1}\right)}=\varepsilon,
\end{aligned}
$$

for every $\omega \in \Omega$. Hence $p \in \operatorname{RF}(T)$.

Remark 4.2. Let $F$ be a nonempty weakly compact and convex subset of a separable Banach space $X$, let $T: \Omega \times F \rightarrow F$ be a continuous asymptotically quasi-nonexpansive random operator with $\sum_{n=1}^{\infty} k_{n}<\infty$ and $(I-T)(\omega, \cdot)$ is demiclosed at zero. Let $\xi_{0}: \Omega \rightarrow F$ be any fixed measurable mapping. Define the sequence of measurable functions $\left\{\xi_{n}\right\}$, $\left\{\eta_{n}\right\},\left\{\varphi_{n}\right\}$, and $\left\{\zeta_{n}\right\}$ from $\Omega$ to $F$ as taken in Theorem 4.1. Then $\left\{\xi_{n}\right\}$ converges to some $p \in \operatorname{RF}(T)$ if and only if there exists some infinite subsequence of $\left\{\xi_{n}\right\}$ which converges to $p \in \operatorname{RF}(T)$. 
TheOREM 4.3. Let $F$ be a nonempty compact and convex subset of a separable uniformly convex Banach space $X$, let $T: \Omega \times F \rightarrow F$ be a continuous $(L-\alpha)$ uniform Lipschitz asymptotically quasi-nonexpansive random operator with $\sum_{n=1}^{\infty} k_{n}<\infty$. Define the sequence of measurable functions $\left\{\xi_{n}\right\},\left\{\eta_{n}\right\},\left\{\rho_{n}\right\}$, and $\left\{\zeta_{n}\right\}$ from $\Omega$ to $F$ as

$$
\begin{gathered}
\xi_{n+1}(\omega)=\alpha_{n} \xi_{n}(\omega)+\beta_{n} T^{n}\left(\omega, \eta_{n}(\omega)\right)+\gamma_{n} \rho_{n}(\omega), \\
\eta_{n}(\omega)=\overline{\alpha_{n}} \xi_{n}(\omega)+\overline{\beta_{n}} T^{n}\left(\omega, \xi_{n}(\omega)\right)+\overline{\gamma_{n}} \zeta_{n}(\omega),
\end{gathered}
$$

where the sequences of numbers $\left\{\alpha_{n}\right\}$ in $[\alpha, 1-\alpha],\left\{\beta_{n}\right\}$ in $[\beta, 1-\beta],\left\{\overline{\alpha_{n}}\right\}$ in $[\alpha, 1],\left\{\overline{\beta_{n}}\right\}$ in $[0, \beta]$, and $\left\{\overline{\gamma_{n}}\right\},\left\{\gamma_{n}\right\}$ in $[0,1]$ satisfy $\alpha_{n}+\beta_{n}+\gamma_{n}=\overline{\alpha_{n}}+\overline{\beta_{n}}+\overline{\gamma_{n}}=1, \lim _{n \rightarrow \infty} \bar{\beta}_{n}=0$, $\sum_{n=1}^{\infty} \gamma_{n}, \sum_{n=1}^{\infty} \overline{\gamma_{n}}<\infty$, for some $\alpha, \beta \in(0,1)$, where $n=1,2, \ldots$. Then $\left\{\xi_{n}\right\}$ converges to some random fixed point of $T$.

Proof. The existence of random fixed point of $T$ follows from Bharucha-Reid's stochastic analogue (see [10]) of well-known Schauder's fixed point theorem. Let $\xi: \Omega \rightarrow F$ be the random fixed point of $T$. Also as proved in Theorem 4.1, we have

$$
\left\|\xi_{n+1}(\omega)-\xi(\omega)\right\| \leq\left(1+k_{n}\right)^{2}\left\|\xi_{n}(\omega)-\xi(\omega)\right\|+m_{n}(\omega)
$$

for every $\omega \in \Omega$. Since, $\sum_{n=1}^{\infty} \gamma_{n}$ and $\sum_{n=1}^{\infty} \overline{\gamma_{n}}<\infty$. Also $\sum_{n=1}^{\infty} k_{n}<\infty$ and $F$ is bounded so $\sum_{n=1}^{\infty} m_{n}(\omega)<\infty$, for every $\omega \in \Omega$. Thus from Lemma 2.9 , we conclude $\lim _{n \rightarrow \infty} \| \xi_{n}(\omega)-$ $\xi(\omega) \|$ exists, for each $\omega \in \Omega$. Consider

$$
\begin{aligned}
\| \eta_{n}(\omega) & -\xi(\omega) \| \\
\leq & \left\|\overline{\alpha_{n}} \xi_{n}(\omega)+\overline{\beta_{n}} T^{n}\left(\omega, \xi_{n}(\omega)\right)+\overline{\gamma_{n}} \zeta_{n}(\omega)-\xi(\omega)\right\| \\
\leq & \overline{\alpha_{n}}\left\|\xi_{n}(\omega)-\xi(\omega)\right\|+\overline{\beta_{n}}\left\|T^{n}\left(\omega, \xi_{n}(\omega)\right)-\xi(\omega)\right\|+\overline{\gamma_{n}}\left\|\zeta_{n}(\omega)-\xi(\omega)\right\| \\
& \leq \overline{\alpha_{n}}\left\|\xi_{n}(\omega)-\xi(\omega)\right\|+\overline{\beta_{n}}\left(1+k_{n}\right)\left\|\xi_{n}(\omega)-\xi(\omega)\right\|+\overline{\gamma_{n}}\left\|\zeta_{n}(\omega)-\xi(\omega)\right\|,
\end{aligned}
$$

for every $\omega \in \Omega$. Thus

$$
\lim \sup _{n \rightarrow \infty}\left\|\eta_{n}(\omega)-\xi(\omega)\right\| \leq \lim \sup _{n \rightarrow \infty}\left\|\xi_{n}(\omega)-\xi(\omega)\right\|=\lim _{n \rightarrow \infty}\left\|\xi_{n}(\omega)-\xi(\omega)\right\|
$$

for every $\omega \in \Omega$. Also

$$
\begin{aligned}
& \lim \sup _{n \rightarrow \infty}\left\|T^{n}\left(\omega, \eta_{n}(\omega)\right)-\xi(\omega)\right\| \\
& \quad \leq \lim \sup _{n \rightarrow \infty}\left(1+k_{n}\right)\left\|\eta_{n}(\omega)-\xi(\omega)\right\| \\
& \quad \leq \lim \sup _{n \rightarrow \infty}\left\|\eta_{n}(\omega)-\xi(\omega)\right\| \leq \lim _{n \rightarrow \infty}\left\|\xi_{n}(\omega)-\xi(\omega)\right\|,
\end{aligned}
$$


for each $\omega \in \Omega$. Finally,

$$
\begin{aligned}
\lim _{n \rightarrow \infty}\left\|\xi_{n+1}(\omega)-\xi(\omega)\right\| & \\
& =\lim _{n \rightarrow \infty}\left\|\alpha_{n} \xi_{n}(\omega)+\beta_{n} T^{n}\left(\omega, \eta_{n}(\omega)\right)+\gamma_{n} \rho_{n}(\omega)-\xi(\omega)\right\| \\
& =\lim _{n \rightarrow \infty}\left\|\alpha_{n}\left(\xi_{n}(\omega)-\xi(\omega)\right)+\beta_{n}\left(T^{n}\left(\omega, \eta_{n}(\omega)\right)-\xi(\omega)\right)+\gamma_{n}\left(\rho_{n}(\omega)-\xi(\omega)\right)\right\| \\
& \left.=\lim _{n \rightarrow \infty} \| \begin{array}{c}
\alpha_{n}\left[\left(\xi_{n}(\omega)-\xi(\omega)\right)+\frac{\gamma_{n}}{2 \alpha_{n}}\left(\rho_{n}(\omega)-\xi(\omega)\right)\right] \\
\beta_{n}\left[\left(T^{n}\left(\omega, \eta_{n}(\omega)\right)-\xi(\omega)\right)+\frac{\gamma_{n}}{2 \beta_{n}}\left(\rho_{n}(\omega)-\xi(\omega)\right)\right.
\end{array}\right] \| \\
& =\lim _{n \rightarrow \infty}\left\|\xi_{n}(\omega)-\xi(\omega)\right\|, \quad \text { for every } \omega \in \Omega .
\end{aligned}
$$

Thus from Lemma 2.8, we have for every $\omega \in \Omega$,

$$
\lim _{n \rightarrow \infty}\left\|\left(\xi_{n}(\omega)-T^{n}\left(\omega, \eta_{n}(\omega)\right)+\left(\frac{\gamma_{n}}{2 \alpha_{n}}-\frac{\gamma_{n}}{2 \beta_{n}}\right)\left(\rho_{n}(\omega)-\xi(\omega)\right)\right]\right\|=0
$$

Since $\gamma_{n} \rightarrow 0$ and $\left\|\rho_{n}(\omega)-\xi(\omega)\right\|$ is bounded, thus

$$
\lim _{n \rightarrow \infty}\left\|\left(\frac{\gamma_{n}}{2 \alpha_{n}}-\frac{\gamma_{n}}{2 \beta_{n}}\right)\left(\rho_{n}(\omega)-\xi(\omega)\right)\right\|=\lim _{n \rightarrow \infty}\left\|\gamma_{n}\left(\frac{1}{2 \alpha_{n}}-\frac{1}{2 \beta_{n}}\right)\right\| \lim _{n \rightarrow \infty}\left\|\rho_{n}(\omega)-\xi(\omega)\right\|,
$$

for each $\omega \in \Omega$. Therefore,

$$
\lim _{n \rightarrow \infty}\left\|\xi_{n}(\omega)-T^{n}\left(\omega, \eta_{n}(\omega)\right)\right\|=0, \quad \text { for each } \omega \in \Omega
$$

Since $F$ is compact, for each $n$, define $G_{n}: \Omega \rightarrow C(X)$ by $G_{n}(\omega)=\operatorname{cl}\left\{\xi_{i}(\omega): i \geq n\right\}$, where cl denotes the closure. Define $G: \Omega \rightarrow C(X)$ by $G(\omega)=\bigcap_{n=1}^{\infty} G_{n}(\omega)$. Then $G$ is measurable and has a measurable selector $\xi$ [31]. We may assume that there exists a subsequence $\left\{\xi_{n_{j}}(\omega)\right\}$ of $\left\{\xi_{n}(\omega)\right\}$ such that for each $\omega \in \Omega$,

$$
\xi_{n_{j}}(\omega) \longrightarrow p(\omega), \quad \text { as } n_{j} \longrightarrow \infty \text {. }
$$

Now using $\gamma_{n_{j}} \rightarrow 0$ and using (4.20), we obtain

$$
\begin{aligned}
& \left\|\xi_{n_{j}+1}(\omega)-\xi_{n_{j}}(\omega)\right\| \\
& \quad \leq\left\|\alpha_{n_{j}} \xi_{n_{j}}(\omega)+\beta_{n_{j}} T^{n_{j}}\left(\omega, \eta_{n_{j}}(\omega)\right)+\gamma_{n_{j}} \rho_{n_{j}}(\omega)-\xi_{n_{j}}(\omega)\right\| \\
& \quad \leq \beta_{n_{j}}\left\|T^{n_{j}}\left(\omega, \eta_{n_{j}}(\omega)\right)-\xi_{n_{j}}(\omega)\right\|+\gamma_{n_{j}}\left\|\rho_{n_{j}}(\omega)-\xi_{n_{j}}(\omega)\right\| \longrightarrow 0,
\end{aligned}
$$


for each $\omega \in \Omega$ when $n_{j} \rightarrow \infty$. Also $\overline{\beta_{n}} \rightarrow 0, \overline{\gamma_{n}} \rightarrow 0,\left\|\zeta_{n}(\omega)-\xi_{n}(\omega)\right\|$ is bounded and using (4.20), we have

$$
\begin{aligned}
\| \eta_{n}(\omega) & -\xi_{n}(\omega) \| \\
= & \left\|\overline{\alpha_{n}} \xi_{n}(\omega)+\overline{\beta_{n}} T^{n}\left(\omega, \xi_{n}(\omega)\right)+\overline{\gamma_{n}} \zeta_{n}(\omega)-\xi_{n}(\omega)\right\| \\
\leq & \overline{\beta_{n}}\left\|T^{n}\left(\omega, \xi_{n}(\omega)\right)-\xi_{n}(\omega)\right\|+\overline{\gamma_{n}}\left\|\zeta_{n}(\omega)-\xi_{n}(\omega)\right\| \longrightarrow 0,
\end{aligned}
$$

for each $\omega \in \Omega$ when $n \rightarrow \infty$. From (4.20) and (4.21), we have

$$
T^{n_{j}}\left(\omega, \eta_{n_{j}}(\omega)\right) \longrightarrow p(\omega), \quad \text { for each } \omega \in \Omega, \text { when } n_{j} \longrightarrow \infty
$$

Now

$$
\begin{aligned}
&\|T(\omega, p(\omega))-p(\omega)\| \\
& \leq\left\|p(\omega)-T^{n_{j}+1}\left(\omega, \eta_{n_{j}+1}(\omega)\right)\right\|+\left\|T^{n_{j}+1}\left(\omega, \eta_{n_{j}+1}(\omega)\right)-T^{n_{j}+1}\left(\omega, \xi_{n_{j}+1}(\omega)\right)\right\| \\
&+\left\|T^{n_{j}+1}\left(\omega, \xi_{n_{j}+1}(\omega)\right)-T^{n_{j}+1}\left(\omega, \xi_{n_{j}}(\omega)\right)\right\| \\
&+\left\|T^{n_{j}+1}\left(\omega, \xi_{n_{j}}(\omega)\right)-T^{n_{j}+1}\left(\omega, \eta_{n_{j}}(\omega)\right)\right\|+\left\|T(\omega, p(\omega))-T^{n_{j}+1}\left(\omega, \eta_{n_{j}}(\omega)\right)\right\| \\
& \leq\left\|p(\omega)-T^{n_{j}+1}\left(\omega, \eta_{n_{j}+1}(\omega)\right)\right\|+L\left\|\eta_{n_{j}+1}(\omega)-\xi_{n_{j}+1}(\omega)\right\|^{\alpha} \\
&+L\left\|\xi_{n_{j}+1}(\omega)-\xi_{n_{j}}(\omega)\right\|^{\alpha}+L\left\|\xi_{n_{j}}(\omega)-\eta_{n_{j}}(\omega)\right\|^{\alpha}+L\left\|T^{n_{j}}\left(\omega, \eta_{n_{j}}(\omega)\right)-p(\omega)\right\|^{\alpha},
\end{aligned}
$$

for each $\omega \in \Omega$. Therefore, $T(\omega, p(\omega))=p(\omega)$, for each $\omega \in \Omega$. The result follows from Remark 4.2.

Remark 4.4. If we take $\gamma_{n}=\overline{\gamma_{n}}=0$ and replace $T^{n}$ by $T$ in Theorem 4.3 , then we obtain Ishikawa random scheme for asymptotically quasi-nonexpansive random operator.

THeOREM 4.5. Let $F$ be a nonempty weakly compact and convex subset of a separable Banach space $X$, let $T: \Omega \times F \rightarrow F$ be a continuous asymptotically quasi-nonexpansive random operator with $\sum_{n=1}^{\infty} k_{n}<\infty$ and $(I-T)(\omega, \cdot)$ is demiclosed at zero. Let $\xi_{0}: \Omega \rightarrow F$ be any fixed measurable mapping. Define the sequence of measurable functions $\left\{\xi_{n}\right\}$ and $\left\{\eta_{n}\right\}$ from $\Omega$ to $F$ as

$$
\begin{gathered}
\xi_{n+1}(\omega)=\alpha_{n} \xi_{n}(\omega)+\beta_{n} T^{n}\left(\omega, \eta_{n}(\omega)\right), \\
\eta_{n}(\omega)=\overline{\alpha_{n}} \xi_{n}(\omega)+\overline{\beta_{n}} T^{n}\left(\omega, \xi_{n}(\omega)\right),
\end{gathered}
$$

where the sequences of numbers $\left\{\alpha_{n}\right\},\left\{\beta_{n}\right\},\left\{\overline{\alpha_{n}}\right\}$, and $\left\{\overline{\beta_{n}}\right\}$ are in $[0,1]$ satisfying $\alpha_{n}+\beta_{n}=$ $\overline{\alpha_{n}}+\overline{\beta_{n}}=1$, for each $n=1,2, \ldots$

Then $\lim _{n \rightarrow \infty}\left\|\xi_{n}(\omega)-\eta(\omega)\right\|=0$ for some $\eta \in \operatorname{RF}(T)$ if for each $\omega \in \Omega, \lim _{n \rightarrow \infty} \| \xi_{n}(\omega)$ $-T\left(\omega, \xi_{n}(\omega)\right) \|=0$ and there exists a constant $L>0$ such that $\| T^{0}\left(\omega, \xi_{n}(\omega)\right)-T(\omega$, $\left.\xi_{n}(\omega)\right) \| \geq L \operatorname{dist}\left(\xi_{n}(\omega),\{\xi(\omega): \xi \in \mathrm{RF}(T)\}\right)$. 
Proof. Note that $\operatorname{RF}(T) \neq \phi$. Since $\left\|\xi_{n}(\omega)-T\left(\omega, \xi_{n}(\omega)\right)\right\| \geq L \operatorname{dist}\left(\xi_{n}(\omega),\{\xi(\omega): \xi \in\right.$ $\operatorname{RF}(T)\})$, and $T\left(\omega, \xi_{n}(\omega)\right) \rightarrow \xi_{n}(\omega)$, for each $\omega \in \Omega$. Therefore, $\lim _{n \rightarrow \infty} \operatorname{dist}\left(\xi_{n}(\omega),\{\xi(\omega)\right.$ : $\xi \in \operatorname{RF}(T)\})=0$, for every $\omega \in \Omega$. This concludes the result using Theorem 4.1 and Remark 4.4.

\section{Acknowledgments}

Authors thank the anonymous referees for perceptive comments.

\section{References}

[1] C. D. Aliprantis and O. Burkinshaw, Principles of Real Analysis, 3rd ed., Academic Press, California, 1998.

[2] J.-P. Aubin and H. Frankowska, Set-Valued Analysis, Systems \& Control: Foundations \& Applications, vol. 2, Birkhäuser Boston, Massachusetts, 1990.

[3] V. H. Badshah and F. Sayyed, Common random fixed points of random multivalued operators on Polish spaces, Indian Journal of Pure and Applied Mathematics 33 (2002), no. 4, 573-582.

[4] I. Beg, Random fixed points of random operators satisfying semicontractivity conditions, Mathematica Japonica 46 (1997), no. 1, 151-155.

[5] _ Approximation of random fixed points in normed spaces, Nonlinear Analysis 51 (2002), no. 8, 1363-1372.

[6] I. Beg and M. Abbas, Equivalence and stability of random fixed point iterative procedures, Journal of Applied Mathematics and Stochastic Analysis 2006 (2006), Article ID 23297, 19 pages.

[7] __ Iterative procedures for solutions of random operator equations in Banach spaces, Journal of Mathematical Analysis and Applications 315 (2006), no. 1, 181-201.

[8] I. Beg and N. Shahzad, Common random fixed points of random multivalued operators on metric spaces, Bollettino della Unione Matematica Italiana 9 (1995), no. 3, 493-503.

[9] A. T. Bharucha-Reid, Random Integral Equations, Mathematics in Science and Engineering, vol. 96, Academic Press, New York, 1972.

[10] _ Fixed point theorems in probabilistic analysis, Bulletin of the American Mathematical Society 82 (1976), no. 5, 641-657.

[11] B. S. Choudhury, Convergence of a random iteration scheme to a random fixed point, Journal of Applied Mathematics and Stochastic Analysis 8 (1995), no. 2, 139-142.

[12] K. M. Das, S. P. Singh, and B. Watson, A note on Mann iteration for quasinonexpansive mappings, Nonlinear Analysis 5 (1981), no. 6, 675-676.

[13] J. B. Diaz and F. T. Metcalf, On the structure of the set of subsequential limit points of successive approximations, Bulletin of the American Mathematical Society 73 (1967), 516-519.

[14] J. Górnicki, Weak convergence theorems for asymptotically nonexpansive mappings in uniformly convex Banach spaces, Commentationes Mathematicae Universitatis Carolinae 30 (1989), no. 2, 249-252.

[15] O. Hanš, Reduzierende zufällige transformationen, Czechoslovak Mathematical Journal 7(82) (1957), 154-158.

[16] _ Random operator equations, Proceedings of the 4th Berkeley Symposium on Mathematical Statistics and Probability, Vol. II, University of California Press, California, 1961, pp. $185-202$.

[17] C. J. Himmelberg, Measurable relations, Fundamenta Mathematicae 87 (1975), 53-72.

[18] S. Itoh, Random fixed-point theorems with an application to random differential equations in $\mathrm{Ba}$ nach spaces, Journal of Mathematical Analysis and Applications 67 (1979), no. 2, 261-273.

[19] S. H. Khan and W. Takahashi, Approximating common fixed points of two asymptotically nonexpansive mappings, Scientiae Mathematicae Japonicae 53 (2001), no. 1, 143-148. 


\section{Convergence of iterative algorithms}

[20] L. S. Liu, Ishikawa and Mann iterative process with errors for nonlinear strongly accretive mappings in Banach spaces, Journal of Mathematical Analysis and Applications 194 (1995), no. 1, 114125.

[21] Q. Liu, Iterative sequences for asymptotically quasi-nonexpansive mappings, Journal of Mathematical Analysis and Applications 259 (2001), no. 1, 1-7.

[22] _ Iterative sequences for asymptotically quasi-nonexpansive mappings with error member, Journal of Mathematical Analysis and Applications 259 (2001), no. 1, 18-24.

[23] _ Iteration sequences for asymptotically quasi-nonexpansive mapping with an error member of uniform convex Banach space, Journal of Mathematical Analysis and Applications 266 (2002), no. $2,468-471$.

[24] Z. Opial, Weak convergence of the sequence of successive approximations for nonexpansive mappings, Bulletin of the American Mathematical Society 73 (1967), 591-597.

[25] N. S. Papageorgiou, Random fixed point theorems for measurable multifunctions in Banach spaces, Proceedings of the American Mathematical Society 97 (1986), no. 3, 507-514.

[26] __ On measurable multifunctions with stochastic domain, Journal of Australian Mathematical Society. Series A 45 (1988), no. 2, 204-216.

[27] R. Penaloza, A characterization of renegotiation proof contracts via random fixed points in Banach spaces, working paper 269, Department of Economics, University of Brasilia, Brasilia, December 2002.

[28] J. Schu, Iterative construction of fixed points of strictly pseudocontractive mappings, Applicable Analysis 40 (1991), no. 2-3, 67-72.

[29] V. M. Sehgal and S. P. Singh, On random approximations and a random fixed point theorem for set valued mappings, Proceedings of the American Mathematical Society 95 (1985), no. 1, 91-94.

[30] N. Shahzad, Random fixed points of $K$-set-and pseudo-contractive random maps, Nonlinear Analysis 57 (2004), no. 2, 173-181.

[31] D. H. Wagner, Survey of measurable selection theorems, SIAM Journal on Control and Optimization 15 (1977), no. 5, 859-903.

Ismat Beg: Department of Mathematics, Centre for Advanced Studies in Mathematics, Lahore University of Management Sciences, Lahore 54792, Pakistan

E-mail address: ibeg@lums.edu.pk

Mujahid Abbas: Department of Mathematics, Centre for Advanced Studies in Mathematics, Lahore University of Management Sciences, Lahore 54792, Pakistan

E-mail address: mujahid@lums.edu.pk 


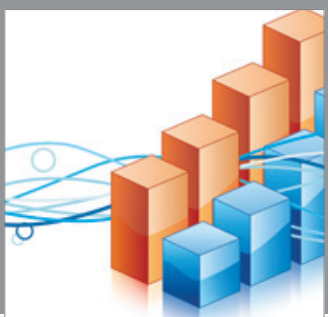

Advances in

Operations Research

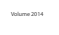

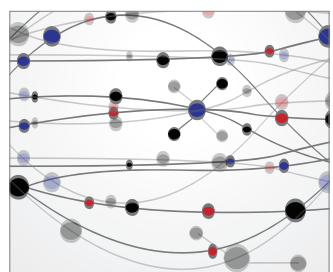

\section{The Scientific} World Journal
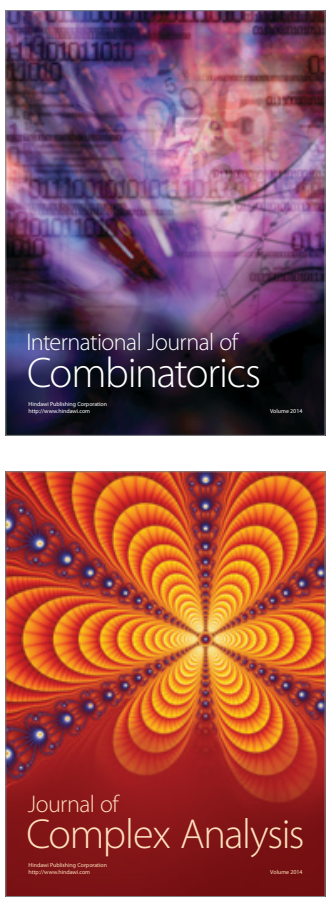

International Journal of

Mathematics and

Mathematical

Sciences
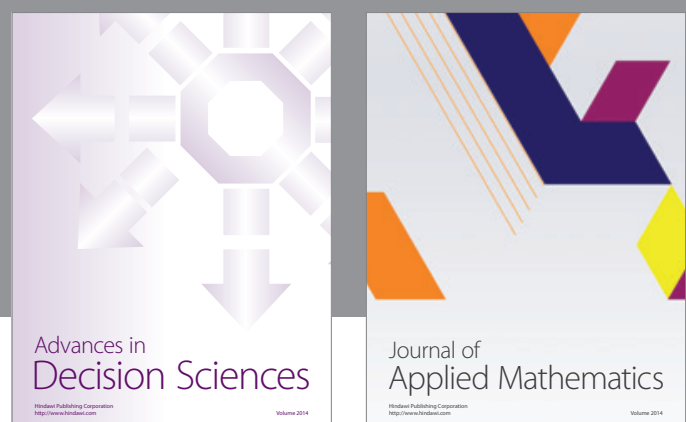

Journal of

Applied Mathematics
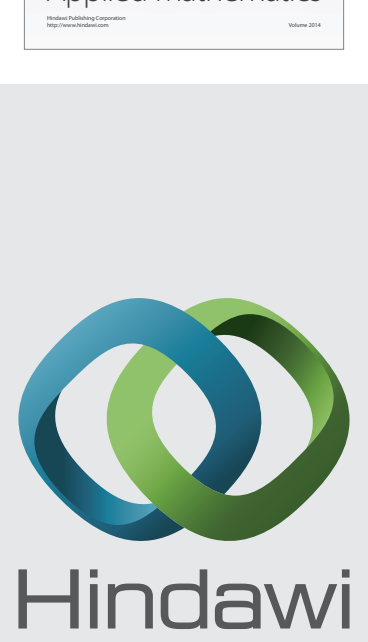

Submit your manuscripts at http://www.hindawi.com
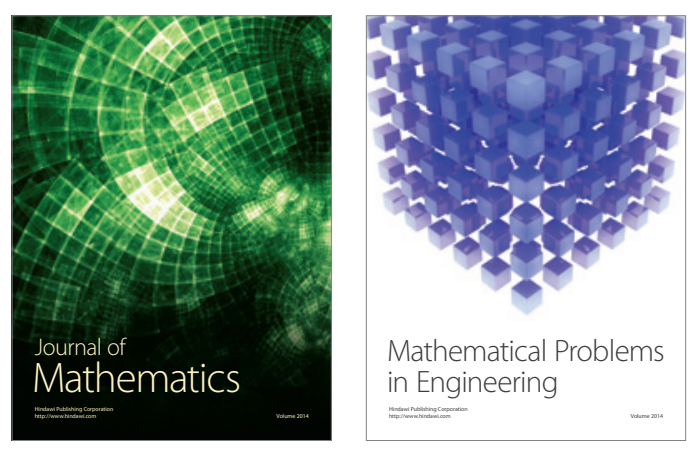

Mathematical Problems in Engineering
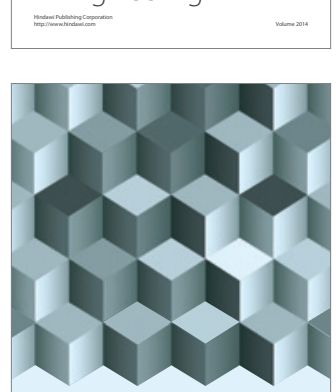

Journal of

Function Spaces
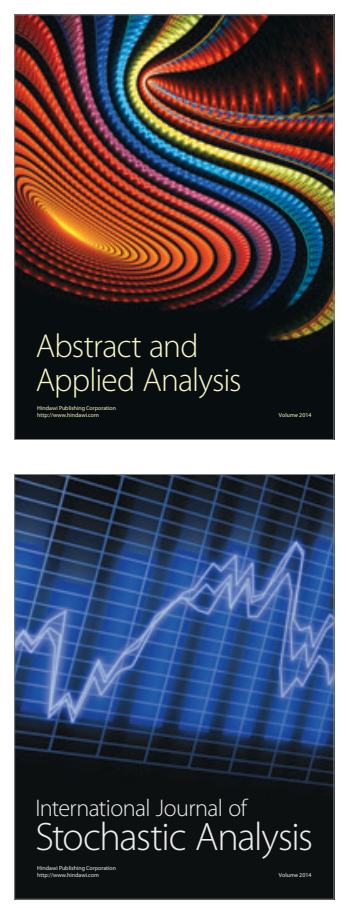

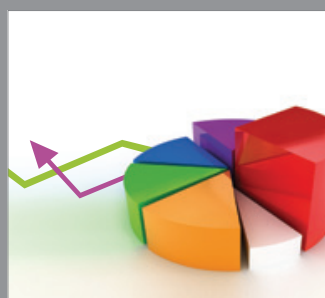

ournal of

Probability and Statistics

Promensencen
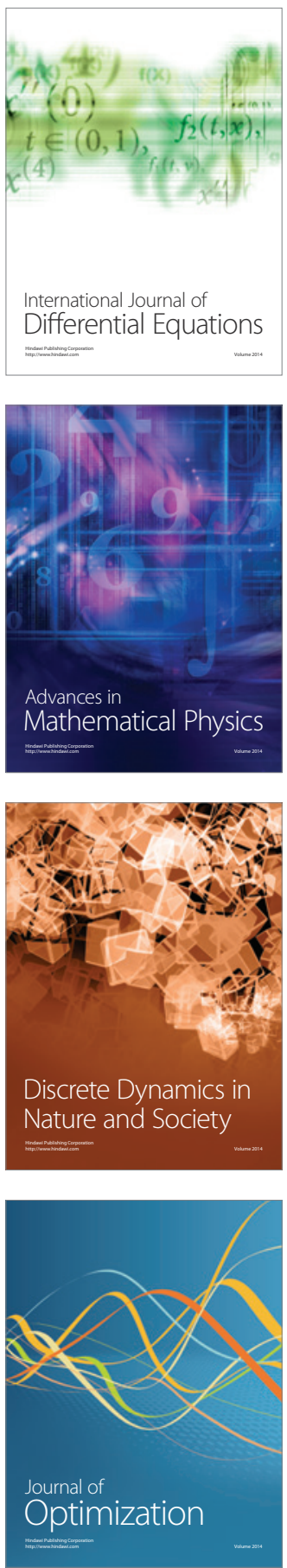